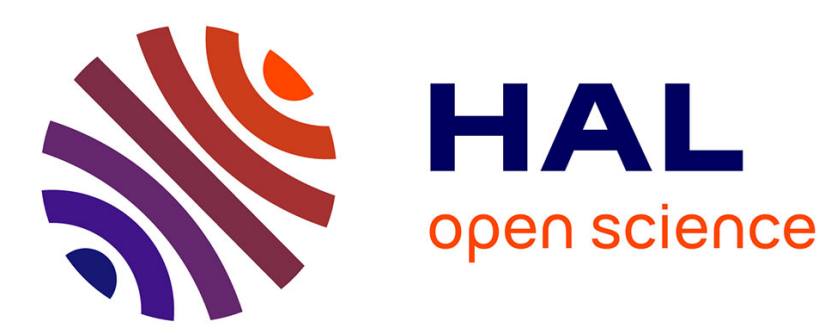

\title{
Perturbed optimization in Banach spaces III: Semi-infinite optimization
}

\author{
J. Frederic Bonnans, Roberto Cominetti
}

\section{To cite this version:}

J. Frederic Bonnans, Roberto Cominetti. Perturbed optimization in Banach spaces III: Semi-infinite optimization. [Research Report] RR-2404, INRIA. 1994. inria-00074271

\section{HAL Id: inria-00074271 \\ https://hal.inria.fr/inria-00074271}

Submitted on 24 May 2006

HAL is a multi-disciplinary open access archive for the deposit and dissemination of scientific research documents, whether they are published or not. The documents may come from teaching and research institutions in France or abroad, or from public or private research centers.
L'archive ouverte pluridisciplinaire HAL, est destinée au dépôt et à la diffusion de documents scientifiques de niveau recherche, publiés ou non, émanant des établissements d'enseignement et de recherche français ou étrangers, des laboratoires publics ou privés. 
Key words. Sensitivity analysis, marginal function, approximate solutions, directional constraint qualification, semi-infinite programming, epilimits

AMS subject classifications. 46N10, 47H19, 49K27, 49K40, 58C $15,90 \mathrm{C} 31$ 


\section{Perturbed optimization in Banach spaces III: Semi-infinite optimization}

J. Frédéric Bonnans, Roberto Cominetti

\section{$\mathbf{N}^{\circ} 2404$}

Décembre 1994

PROGRAMME 5

Traitement du signal,

automatique

et productique

\section{apport \\ de recherche}





\title{
Perturbed optimization in Banach spaces III: Semi-infinite optimization
}

\author{
J. Frédéric Bonnans* , Roberto Cominetti** \\ Programme 5 - Traitement du signal, automatique et productique \\ Projet Programmation mathématique
}

Rapport de recherche $\mathrm{n}^{\circ} 2404$ - Décembre 1994 - 17 pages

\begin{abstract}
This paper is devoted to the study of perturbed semi-infinite optimization problems, i.e. minimization over $\mathbb{R}^{n}$ with an infinite number of inequality constraints. We obtain the second order expansion of the optimal value function and the first order expansion of approximate optimal solutions in two cases: (i) when the number of binding constraints is finite, and (ii) when the inequality constraints are parametrized by a real scalar.

These results are partly obtained by specializing the sensitivity theory for perturbed optimization developed in part I ( $c f$. [4]), and deriving specific sharp lower estimates for the optimal value function which take into account the curvature of the positive cone in the space $C(\Omega)$ of continuous real-valued functions.
\end{abstract}

(Résumé : tsvp)

* Email: Frederic.Bonnans@inria.fr

** Universidad de Chile, Casilla 170/3 Correo 3, Santiago, Chile. Partially supported by Fondecyt 1940564 


\section{Optimisation avec perturbation dans les espaces de Banach III : Optimization semi infinie ${ }^{* * *}$}

Résumé : Cet article est consacré à l'étude de problèmes d'optimization semi infinie (minimisation sur $\mathbb{R}^{n}$ avec un nombre infini de contraintes) avec perturbation. Nous obtenons le développement au deuxième ordre du coût, et au premier ordre des solutions approchées, dans deux cas : (i) quand le nombre de contraintes actives est fini, et (ii) quand les contraintes d'inégalité sont paramétrées par un nombre réel.

Ces résultats sont en partie obtenus en spécialisant la théorie de la sensibilité pour l'optimisation avec perturbation développée dans la partie I ( $c f$. [4]), et en obtenant des estimations inférieures spécifiques fortes du coût, qui prennent en compte la courbure du cône positif de l'espace $C(\Omega)$ des fonctions continues à valeurs réelles. 
1. Introduction. This paper is the last of a trilogy devoted to the analysis of parametric optimization problems of the form

$$
\min _{x}\{f(x, u): G(x, u) \in K\}
$$

with $X$ and $Y$ Banach spaces, $K$ a closed convex subset of $Y$, and $f(x, u), G(x, u)$ mappings of class $\mathcal{C}^{2}$ from $X \times \mathbb{R}$ into $\mathbb{R}$ and $Y$ respectively. This third part is devoted to the study of the parametric semi-infinite optimization problem:

$$
\min _{x}\left\{f(x, u): G(x, u)_{\omega} \geq 0, \forall \omega \in \Omega\right\}
$$

where $\Omega$ is a compact metric space, $G(x, u):=\left\{G(x, u)_{\omega}\right\}_{\omega \in \Omega}$ belongs to $C(\Omega)$, the space of continuous functions on $\Omega$ endowed with the max norm, and the mapping $(x, u) \rightarrow$ $(f(x, u), G(x, u))$ is of class $\mathcal{C}^{2}$ from $\mathbb{R}^{n} \times \mathbb{R}^{+}$into $\mathbb{R} \times C(\Omega)$. Since

$$
C_{+}(\Omega):=\{y \in C(\Omega): y \geq 0\}
$$

is a closed convex cone in the Banach space $C(\Omega)$, it follows that $\left(P_{u}\right)$ is a particular case of the above abstract optimization problem.

Semi-infinite optimization problems occur in robust control theory, the design of filters, the design of devices having to respect some specifications in a certain range of pressure and temperature, as well as in optimal control problems when the control has a finite dimensional parametrization; see [16]. However, the wealth of applications is not the only motivation for studying semi-infinite optimization. In the last years, a rather complete perturbation theory has been developed for optimization problems with a finite number of constraints, the so-called perturbed nonlinear programming problem, e.g. [2],[6],[8],[19]. The theory of perturbed semi-infinite optimization problems, although it seems much easier than the general perturbation problem in Banach space, includes an essential difficulty related to the curvature of $C_{+}(\Omega)$. As a consequence, the standard second-order upper- and underestimates for the cost do not, in general, coincide. Our main contribution here is to exhibit a sharp under-estimate that, in some cases, is equal to the parabolic upper-estimate.

There exists already a large body of literature on semi-infinite optimization. See the early references [3] and [11]. The recent review [9] describes in particular the so-called reduction theory that reduces $\left(P_{u}\right)$ to an optimization problem with a finite number of constraints (see also [13]). This reduction is possible when the contact set includes a finite number of points and each of them can be expressed locally as a function of the data, typically a local solution of an optimization problem with finitely many constraints. Then the perturbation theory for nonlinear programming can be applied for deriving optimality conditions, as well as for conducting a perturbation analysis; see the early reference [18].

In this paper we do not use any reduction device. In this way we may handle some cases where there exists a continuum of binding constraints, specially when $\Omega$ is a one-dimensional interval. We are also able to treat the case of a finite number of binding constraints in cases where the reduction theory does not apply. 
The paper is organized as follows. In $\S 2$ we discuss the directional qualification condition introduced in part I. We characterize it and show how to deduce a first order upper estimate. Section 3 is devoted to the parabolic (second order) upper estimates. There we combine the technique of parabolic estimates with the directional qualification condition and a characterization of second order tangent sets to $C_{+}(\Omega)$, recently obtained in [7]. This upper estimate combined with the strong quadratic growth condition implies the upper-Lipschitz property for the set of solutions. In $\S 4$ we discuss some sharp lower estimates. We use there specific properties of semi-infinite optimization, among them the fact that an extremal multiplier has a finite support. Then in $\S 5$ we recapitulate and state our main result.

2. Directional qualification. We start with some notations. The feasible set, value function, and set of solutions of $\left(P_{u}\right)$ are denoted as

$$
\begin{aligned}
F(u) & :=\left\{x \in \mathbb{R}^{n}: G(x, u) \geq 0\right\}, \\
v(u) & :=\inf \{f(x, u): x \in F(u)\}, \\
S(u) & :=\{x \in F(u): f(x, u)=v(u)\} .
\end{aligned}
$$

Similarly, given any optimization problem $(P)$, we define $F(P), v(P)$ and $S(P)$ as the feasible set, value function, and set of solutions of $(P)$.

We recall that the dual space of $C(\Omega)$ is the set $M(\Omega)$ of bounded measures, see e.g. [22]. If $(\lambda, y) \in M(\Omega) \times C(\Omega)$ then $\langle\lambda, y\rangle=\int_{\Omega} y(\omega) d \lambda(\omega)$. The support of $\lambda \in M(\Omega)$, denoted by $\operatorname{supp}(\lambda)$, is defined as the complement of the greatest open subset of $\Omega$ over which $|\lambda|$ is null. The negative cone of $M(\Omega)$ is denoted $M_{-}(\Omega)$.

The Lagrangian function associated with $\left(P_{u}\right)$ is

$$
\mathcal{L}(x, \lambda, u)=f(x, u)+\int_{\Omega} G(x, u)_{\omega} d \lambda(\omega)
$$

With $x \in F(u)$ we associate the set of Lagrange multipliers

$$
\Lambda_{u}(x):=\left\{\lambda \in M_{-}(\Omega): \operatorname{supp}(\lambda) \subset Z(G(x, u)) \text { and } \mathcal{L}_{x}^{\prime}(x, \lambda, u)=0\right\}
$$

where, for $y \in C(\Omega)$, the set $Z(y)$ is the contact set defined as

$$
Z(y):=\{\omega \in \Omega: y(\omega)=0\} .
$$

Let us fix a particular solution $x_{0} \in S(0)$ and let us denote $\Lambda_{0}:=\Lambda_{0}\left(x_{0}\right)$ and $Z_{0}:=$ $Z\left(G\left(x_{0}, 0\right)\right)$. The problem with linearized data $(L)$ and its dual $(D)$ are

$$
\begin{gathered}
\min _{d}\left\{f^{\prime}\left(x_{0}, 0\right)(d, 1): G^{\prime}\left(x_{0}, 0\right)(d, 1) \geq 0 \text { on } Z_{0}\right\}, \\
\max _{\lambda \in \Lambda_{0}} \mathcal{L}_{u}^{\prime}\left(x_{0}, \lambda, 0\right) .
\end{gathered}
$$

We consider the directional qualification hypothesis

$$
\exists \hat{d} \in \mathbb{R}^{n}: G^{\prime}\left(x_{0}, 0\right)(\hat{d}, 1)>0 \text { on } Z_{0}
$$


which is to be compared to the standard qualification hypothesis (see [12])

$$
\exists \tilde{d} \in \mathbb{R}^{n}: G_{x}^{\prime}\left(x_{0}, 0\right) \tilde{d}>0 \text { on } Z_{0} .
$$

The following is essentially known.

LEMMa 2.1. Condition $(C Q)$ is equivalent to each of the two conditions below:

(i) There exists $\tilde{d} \in \mathbb{R}^{n}$ such that

$$
G\left(x_{0}, 0\right)+G_{x}^{\prime}\left(x_{0}, 0\right) \tilde{d}>0 \text { on } \Omega .
$$

(ii) The set $\Lambda_{0}$ is non empty and bounded.

Proof. The equivalence between $(C Q)$ and $(i)$ is proved in [20]. That $(C Q)$ implies $(i i)$ follows from [23]. Let us prove that (ii) implies $(C Q)$. If $(C Q)$ does not hold, then the following linear semi-infinite optimization problem

$$
\min _{d, z}\left\{z: G^{\prime}\left(x_{0}, 0\right)_{\omega}(d, 0)+z \geq 0, \forall \omega \in Z_{0}\right\}
$$

has value 0 . It follows that $(d, z)=(0,0)$ is a solution of this problem at which the qualification condition is satisfied by the direction $(0,1)$. By $(i) \Rightarrow(i i)$, there exists at least one multiplier $\hat{\lambda}$. Expressing the optimality conditions, we find that $\hat{\lambda} \in M_{-}(\Omega) \backslash\{0\}$, $\operatorname{supp}(\hat{\lambda}) \subset Z_{0}$, and $\hat{\lambda} \circ G_{x}^{\prime}\left(x_{0}, 0\right)=0$. It follows that whenever $\lambda \in \Lambda_{0}$, and $t \in \mathbb{R}_{+}$, then $\lambda+t \hat{\lambda} \in \Lambda_{0}$, in contradiction with (ii).

A similar result holds for condition $(D C Q)$.

Lemma 2.2. Condition $(D C Q)$ is equivalent to

(i) There exist $\varepsilon>0$ and $\tilde{d} \in \mathbb{R}^{n}$ such that

$$
G\left(x_{0}, 0\right)+\varepsilon G^{\prime}\left(x_{0}, 0\right)(\tilde{d}, 1)>0 \text { on } \Omega .
$$

If in addition $\Lambda_{0} \neq \emptyset$, then $(D C Q)$ is equivalent to

(ii) The set $S(D)$ is non empty and bounded.

Proof. Noting that $(D C Q)$ is nothing but the standard qualification condition for the set of constraints $\{G(x, u) \geq 0 ; u \geq 0\}$, and applying Lemma 2.1, we obtain the equivalence of $(D C Q)$ and $(i)$.

Assume now that $\Lambda_{0} \neq \emptyset$. That ( $D C Q$ ) implies (ii) follows from [4, Proposition 3.1]. Conversely, if $(D C Q)$ does not hold we have

$$
\alpha:=\min _{d, z}\left\{z: G^{\prime}\left(x_{0}, 0\right)_{\omega}(d, 1)+z \geq 0, \forall \omega \in Z_{0}\right\} \geq 0 .
$$

Considering the perturbation function

$$
\varphi((d, z), h)=\left\{\begin{array}{cl}
z & \text { if } h(\omega)+G^{\prime}\left(x_{0}, 0\right)_{\omega}(d, 1)+z \geq 0, \forall \omega \in Z_{0} \\
+\infty & \text { otherwise }
\end{array}\right.
$$


we obtain the dual problem

$$
\min _{\lambda}\left\{-\int_{\Omega} G_{u}^{\prime}\left(x_{0}, 0\right) d \lambda: \lambda \in M_{-}(\Omega), \operatorname{supp}(\lambda) \subset Z_{0}, \int_{\Omega} d \lambda=-1, \lambda \circ G_{x}^{\prime}\left(x_{0}, 0\right)=0\right\} .
$$

Applying [4, Thm. A2] we get the existence of an optimal solution $\hat{\lambda}$ for this latter problem, and we have

$$
\int_{\Omega} G_{u}^{\prime}\left(x_{0}, 0\right) d \hat{\lambda}=\alpha \geq 0 .
$$

It follows that for each $\lambda \in S(D)$ and every $t>0$ we have $\lambda+t \hat{\lambda} \in S(D)$, contradicting the boundedness of $S(D)$ stated in $(i i)$.

From the above Lemma and ([5], Propsition 5.2), it follows that $(D C Q)$ is a particular case of the abstract directional constraint qualification of part I.

Proposition 2.3. If (DCQ) holds, then

$$
\limsup _{u \downarrow 0} \frac{v(u)-v(0)}{u} \leq v(D)=v(L) .
$$

Proof. This follows from Propositions 2.1 and 3.1 in [4], and Lemma 2.2 above.

Remark. The above statements hold when $f$ and $G$ are merely of class $\mathcal{C}^{1}$.

3. Second order upper estimates. Define a path as a mapping $u \rightarrow x_{u}$ from $\mathbb{R}_{+}$to $X$, with $x_{u} \rightarrow x_{0}$ when $u \downarrow 0$. The path is said to be feasible if $G\left(x_{u}, u\right) \in K$ for $u$ small enough. In the study of second order upper estimates, we analyze feasible paths of the form

$$
x_{u}:=x_{0}+u d+\frac{u^{2}}{2} z+o\left(u^{2}\right) .
$$

Feasibility of $x_{u}$ implies some relations between the expansion of $G\left(x_{u}, u\right)$ and the geometry of $C_{+}(\Omega)$. Given a convex subset $K$ of a Banach space $Y$, we define the first order tangent set at $y \in K$ as

$$
T_{K}(y):=\{h \in Y: \text { there exists } o(t) \text { such that } y+t h+o(t) \in K\} .
$$

Similarly, the second order tangent set at $y \in K$ in the direction $h \in T(y)$ is

$$
T_{K}^{2}(y, h):=\left\{z \in Y: \text { there exists } o\left(t^{2}\right) \text { such that } y+t h+\frac{t^{2}}{2} z+o\left(t^{2}\right) \in K\right\} .
$$

For the sake of simplicity we write $T:=T_{C_{+}(\Omega)}$ and $T^{2}:=T_{C_{+}(\Omega)}^{2}$, and we denote the terms of the second-order expansion of $f\left(x_{u}, u\right)$ and $G\left(x_{u}, u\right)$ as

$$
\begin{aligned}
\Psi_{f}(z, d) & :=f_{x}^{\prime}\left(x_{0}, 0\right) z+f^{\prime \prime}\left(x_{0}, 0\right)(d, 1)(d, 1), \\
\Psi_{G}(z, d) & :=G_{x}^{\prime}\left(x_{0}, 0\right) z+G^{\prime \prime}\left(x_{0}, 0\right)(d, 1)(d, 1) .
\end{aligned}
$$


Expanding $G\left(x_{u}, u\right)$ we obtain that if $x_{u}$ is a feasible path, then

$$
\begin{gathered}
G^{\prime}\left(x_{0}, 0\right)(d, 1) \in T\left(G\left(x_{0}, 0\right)\right) \\
\Psi_{G}(z, d) \in T^{2}\left(G\left(x_{0}, 0\right), G^{\prime}\left(x_{0}, 0\right)(d, 1)\right)
\end{gathered}
$$

and, when $d \in S(L)$, we get

$$
v(u) \leq v(0)+u v(L)+\frac{u^{2}}{2} \Psi_{f}(z, d)+o\left(u^{2}\right) .
$$

In [4], it was shown that an upper estimate of the second order variation of the cost is obtained by minimizing $\Psi_{f}(z, d)$ over those $z$ satisfying (2). The purpose of this section is to make explicit this bound in the case of semi-infinite programming. In the statement of our result, we use some expressions for the tangent sets of $K=C_{+}(\Omega)$. The first order tangent cone is well known, see e.g. [20]:

$$
T(y)=\{h \in C(\Omega): h \geq 0 \text { on } Z(y)\} .
$$

In particular, the tangent cone at $G\left(x_{0}, 0\right)$ is

$$
T\left(G\left(x_{0}, 0\right)\right)=\left\{h \in C(\Omega): h \geq 0 \text { on } Z_{0}\right\} .
$$

A formula for the second order tangent set has been recently obtained by [7]. This formula uses the concept of lower-epilimit that we recall now, referring to [1] for a detailed exposition. Let $\left(A_{t}\right)_{t>0}$ be a family of subsets of a Banach space $Y$. The upper limit of $\left(A_{t}\right)_{t>0}$ at $t=0$ in the sense of Painlevé-Kuratowski is defined as

$$
\limsup _{t \downarrow 0} A_{t}:=\left\{y \in Y: \liminf _{t \downarrow 0} d\left(y, A_{t}\right)=0\right\} .
$$

The lower-epilimit of a family $\left(f_{t}\right)_{t>0}$ of extended real valued functions on the topological space $K$ is defined as the function whose epigraph is $\lim \sup _{t \downarrow 0}$ epi $f_{t}$, where

$$
\text { epi } f_{t}:=\left\{(x, r) \in K \times \mathbb{R}: f_{t}(x) \leq r\right\}
$$

is the epigraph of $f_{t}$. An alternative characterization is given by

$$
\text { e-liminf } f_{t}(x)=\sup _{V \in \mathcal{N}(x)} \liminf _{t \downarrow 0} \inf _{y \in V} f_{t}(y)=\liminf _{(t, y) \rightarrow(0+, x)} f_{t}(y),
$$

where $\mathcal{N}(x)$ is the set of neighborhoods of $x$.

Proposition 3.1. (cf. [7]) Let $y \in C_{+}(\Omega)$ and $h \in T(y)$. Then

$$
T^{2}(y, h)=\{h \in C(\Omega): h+\tau(y, h) \geq 0\},
$$


where $\tau(f, v)$ is the l.s.c. extended real valued function defined by

$$
\tau(f, v):=\mathrm{e}-\liminf _{t \downarrow 0}\left[\frac{f+t v}{t^{2} / 2}\right] .
$$

Equivalently, $\tau(f, v)$ is given by the formula:

$$
\tau(f, v)(\omega)=\left\{\begin{array}{cl}
0 & \text { if } \omega \in \operatorname{int} Z(f) \text { and } v(\omega)=0 \\
-\theta(\omega) & \text { if } \omega \in \operatorname{bd} Z(f) \text { and } v(\omega)=0 \\
+\infty & \text { otherwise }
\end{array}\right.
$$

where

$$
\theta(\omega):=\limsup _{\substack{y \rightarrow \omega \\ f(y)>0}} \frac{[-v(y)]_{+}^{2}}{2 f(y)} .
$$

In view of this result, defining

$$
\begin{aligned}
\Xi(d, u)_{\omega} & :=\frac{G\left(x_{0}, 0\right)_{\omega}+u G^{\prime}\left(x_{0}, 0\right)_{\omega}(d, 1)}{u^{2} / 2}, \\
\tau_{d}(\omega) & :=\mathrm{e}-\liminf _{u \downarrow 0} \Xi(d, u)_{\omega},
\end{aligned}
$$

we get the characterization

$$
T^{2}\left(G\left(x_{0}, 0\right) ; G^{\prime}\left(x_{0}, 0\right)(d, 1)\right)=\left\{h \in C(\Omega): h+\tau_{d} \geq 0\right\} .
$$

Writing in short $T^{2}(d)$ for the above set, we see that $T^{2}(d) \neq \phi$ if and only if $\tau_{d}>-\infty$. In such a case the support function of $T^{2}(d)$ can be characterized as

$$
\sigma\left(\lambda, T^{2}(d)\right):=\sup \left\{\int_{\Omega} h(\omega) d \lambda(\omega): h \in T^{2}(d)\right\}=-\int_{\Omega} \tau_{d}(\omega) d \lambda(\omega)
$$

for all $\lambda \in S(D)$ (since $\lambda \leq 0$ and $\tau_{d}$ is l.s.c. and nonpositive on $\operatorname{supp}(\lambda)$, the integral on the right hand side above is well defined). To make this equality always valid we define $\int_{\Omega} \tau_{d}(\omega) d \lambda(\omega):=+\infty$ whenever $\tau_{d}$ takes the value $-\infty$.

The function $-\tau_{d}$ may be interpreted as an upper-curvature function.

Given $d \in S(L)$, relations (1)-(3) suggest to consider the subproblem

$$
\min _{z}\left\{\Psi_{f}(z, d): \Psi_{G}(z, d)+\tau_{d} \geq 0\right\}
$$

with which we associate a dual formulation

$$
\max _{\lambda \in S(D)} \mathcal{L}^{\prime \prime}\left(x_{0}, \lambda, 0\right)(d, 1)(d, 1)+\int_{\Omega} \tau_{d}(\omega) d \lambda(\omega) .
$$


We also introduce the problem

$$
\min \left\{v\left(L_{d}\right): d \in S(L)\right\} .
$$

Whenever $v^{\prime}(0)$ exists, we define the upper and lower second-order Dini derivatives

$$
\begin{aligned}
& v_{+}^{\prime \prime}(0):=\limsup _{u \downarrow 0} 2\left[v(u)-v(0)-u v^{\prime}(0)\right] / u^{2}, \\
& v_{-}^{\prime \prime}(0):=\liminf _{u \downarrow 0} 2\left[v(u)-v(0)-u v^{\prime}(0)\right] / u^{2} .
\end{aligned}
$$

Proposition 3.2. If $(D C Q)$ holds, then $v\left(L_{d}\right)=v\left(D_{d}\right)$ for all $d \in S(L)$, and we have $v(Q)<+\infty$ iff there exists $d \in S(L)$ such that $\tau_{d}(\omega)>-\infty$ for all $\omega \in \Omega$. Moreover, if $S(L)$ is nonempty and $v(Q)>-\infty$, then we have

$$
v(u) \leq v(0)+u v(L)+\frac{u^{2}}{2} v(Q)+o\left(u^{2}\right) .
$$

In particular, if there exists $v^{\prime}(0)=v(L)$, we get

$$
v_{+}^{\prime \prime}(0) \leq \inf _{d \in S(L)} \max _{\lambda \in S(D)}\left\{\mathcal{L}^{\prime \prime}\left(x_{0}, \lambda, 0\right)(d, 1)(d, 1)+\int_{\Omega} \tau_{d}(\omega) d \lambda(\omega)\right\}
$$

Proof. This is a consequence of Propositions 2.1, 2.2 and 4.2 in [4].

By the above Proposition, $v(Q)<+\infty$ iff $\tau_{d}>-\infty$ for some $d \in S(L)$. We show that a sufficient condition for this is a quadratic growth condition, recently introduced in [20].

Lemma 3.3. Suppose that $G^{\prime}\left(x_{0}, 0\right)$ is Lipschitz w.r.t. $\omega$ and assume that $G\left(x_{0}, 0\right)$ satisfies the quadratic growth condition

$$
\exists c>0 \text { s.t. } G\left(x_{0}, 0\right)_{\omega} \geq c \operatorname{dist}\left(\omega, Z_{0}\right)^{2} .
$$

Then $\tau_{d}>-\infty$ for all $d \in S(L)$.

Proof. Using (4) it suffices to show that for all $\omega \in \mathrm{bd} Z_{0}$ with $G^{\prime}\left(x_{0}, 0\right)(d, 1)_{\omega}=0$ we have $\theta(\omega)<+\infty$. To this end let $L$ be a Lipschitz constant for $\omega \rightarrow G^{\prime}\left(x_{0}, 0\right)(d, 1)_{\omega}$. For each $\omega \notin Z_{0}$ let $\omega_{0}$ be a projection of $\omega$ onto $Z_{0}$. Then $\omega_{0}$ lies on the boundary of $Z_{0}$ so that $G\left(x_{0}, 0\right)_{\omega_{0}}=0$, and since $G^{\prime}\left(x_{0}, 0\right)(d, 1) \in T\left(G\left(x_{0}, 0\right)\right)$ we deduce $G^{\prime}\left(x_{0}, 0\right)(d, 1)_{\omega_{0}} \geq 0$. We obtain

$$
-G^{\prime}\left(x_{0}, 0\right)(d, 1)_{\omega} \leq-G^{\prime}\left(x_{0}, 0\right)(d, 1)_{\omega_{0}}+L d\left(\omega, w_{0}\right) \leq L \operatorname{dist}\left(\omega, Z_{0}\right) .
$$

From this and $(Q G C)$ we deduce

$$
\left[-G^{\prime}\left(x_{0}, 0\right)(d, 1)_{\omega}\right]_{+}^{2} \leq L^{2} \operatorname{dist}\left(\omega, Z_{0}\right)^{2} \leq \frac{L^{2}}{c} G\left(x_{0}, 0\right)_{\omega}
$$

which implies $\theta(\omega) \leq L^{2} /(2 c)<+\infty$. $\square$

Remark. Assuming $(D C Q)$, we know by Lemma 2.2 that $S(D)$ is bounded. Let $d \in$ $S(L)$. If $(Q G C)$ holds, as a consequence of the bound established for $\theta(\omega)$, the amount $\int_{\Omega} \tau_{d}(\omega) d \lambda(\omega)$ is bounded uniformly for $\lambda \in S(D)$, so that $v\left(D_{d}\right)$ is finite. 
4. Stability of solutions. We state next a sufficient condition for ensuring a Lipschitz behavior of the (sub) optimal paths $x_{u}$ of the perturbed problems. The result, which is a rather straightforward application of what precedes and [4, Proposition 6.3], is based on the strong second order sufficient condition

$$
\max _{\lambda \in S(D)} \mathcal{L}_{x}^{\prime \prime}\left(x_{0}, \lambda, 0\right) d d>0 \text { for all } d \text { in } C \backslash\{0\},
$$

where $C$ denotes the critical cone

$$
C:=\left\{d \in \mathbb{R}^{n}: f_{x}^{\prime}\left(x_{0}, 0\right) d \leq 0 \text { and } G_{x}^{\prime}\left(x_{0}, 0\right) d \geq 0 \text { on } Z_{0}\right\} .
$$

Proposition 4.1. Suppose that

(i) $(D C Q)$ holds and $\Lambda_{0} \neq \emptyset$.

(ii) There exists $d \in S(L)$ such that $\tau_{d}(\omega)>-\infty, \forall \omega \in \Omega$.

(iii) (SOC) holds.

Then every $O\left(u^{2}\right)$-optimal path $x_{u}$ satisfies $x_{u}=x_{0}+O(u)$.

Remark. Combining Lemma 3.3 and Proposition 4.1, we obtain a sufficient condition for Lipschitz behavior of solutions, similar to the result of [20].

Remark. Following [10], we may introduce the strong quadratic growth condition

$$
\exists \alpha>0, c>0 ; F(x, u) \geq v(0)+u v(L)+\alpha \operatorname{dist}\left(x, S_{0}\right)^{2}-c u^{2},
$$

where

$$
F(x, u):=\left\{\begin{array}{ll}
f(x, u) & \text { if } \\
+\infty & \text { if not. }
\end{array} \quad G(x, u) \geq 0,\right.
$$

Then we can show that $(S O C) \Rightarrow(S Q G)$, and Proposition 4.1 is still valid if we replace assumption (iii) by $(S Q G)$.

5. Lower estimates. We recall for reference the following standard lower estimate, which is in fact a particular case of the general lower estimate of part I.

Lemma 5.1. Let us assume $(D C Q)$ and suppose that there exists a path of o $\left(u^{2}\right)$-optimal solutions $x_{u}$ satisfying $x_{u}=x_{0}+O(u)$. Then $\Lambda_{0} \neq \emptyset, v^{\prime}(0)$ exists with $v^{\prime}(0)=v(L)=v(D)$, $S(L) \neq \emptyset$, and we have

$$
v_{-}^{\prime \prime}(0) \geq \inf _{d \in S(L)} \max _{\lambda \in S(D)} \mathcal{L}^{\prime \prime}\left(x_{0}, \lambda, 0\right)(d, 1)(d, 1) .
$$

Proof. The existence of $v^{\prime}(0)$ as well as the equality $v^{\prime}(0)=v(L)=v(D)$ follows from [4, Proposition 3.2]. The finiteness of $v^{\prime}(0)=v(D)$ implies that $\Lambda_{0} \neq \emptyset$, and [4, Proposition 3.3] gives $S(L) \neq \emptyset$. The lower estimate on $v_{-}^{\prime \prime}(0)$ is then obtained by applying [4, Proposition 4.3(b)]. 
Comparing the previous lower estimate to the upper estimate (9) in $\S 3$, we observe a gap due to the curvature of $C_{+}(\Omega)$ at $G\left(x_{0}, 0\right)$. More precisely, if

$$
\tau_{d}=0 \text { on } \operatorname{supp}(\lambda), \forall \lambda \in S(D),
$$

then the two estimates coincide but, as one can see from (4) and (5), this may only occur in some very special situations.

We are then led to search for sharper lower estimates. We will obtain a lower estimate on $v_{-}^{\prime \prime}(0)$ involving the upper-epilimit of $\Xi(d, u)$.

We recall the concept of upper-epilimit, for which we refer again to [1]. Let $\left(A_{t}\right)_{t>0}$ be a family of subsets of a Banach space $Y$. The lower limit of $\left(A_{t}\right)_{t>0}$ at $t=0$ in the sense of Painlevé-Kuratowski is defined as

$$
\liminf _{t \downarrow 0} A_{t}:=\left\{y \in Y: \limsup _{t \downarrow 0} d\left(y, A_{t}\right)=0\right\} .
$$

The upper-epilimit of a family $\left(f_{t}\right)_{t>0}$ of extended real valued functions on the topological space $K$ is defined as the function whose epigraph is $\liminf _{t \downarrow 0}$ epi $f_{t}$. A useful formula is

$$
\text { e- } \limsup _{t \downarrow 0} f_{t}(x)=\sup _{V \in \mathcal{N}(x)} \limsup _{t \downarrow 0} \inf _{y \in V} f_{t}(y)
$$

When the upper and lower epilimits coincide at a given point, we shall say that the family of functions epiconverges at that point, and we shall denote

$$
\text { e- } \lim _{t \downarrow 0} f_{t}(x)=\mathrm{e}-\liminf _{t \downarrow 0} f_{t}(x)=\mathrm{e}-\limsup _{t \downarrow 0} f_{t}(x) .
$$

We shall say that the family of functions epiconverges on a subset $K_{0} \subset K$ if it epiconverges at each point of $K_{0}$.

The next Proposition makes use of the set of extreme points of $S(D)$, which will be denoted $S^{*}(D)$. The result will be derived under a technical assumption $\left(H_{\hat{\omega}}\right)$ which will be further clarified afterwards. $B(\hat{\omega}, r)$ denotes the ball of center $\hat{\omega}$ and radius $r$.

Proposition 5.2. Assume (DCQ) and suppose that there exists an o( $\left.u^{2}\right)$-optimal path such that $x_{u}=x_{0}+O(u)$. Let $G\left(x_{0}, 0\right), G^{\prime}\left(x_{0}, 0\right), G^{\prime \prime}\left(x_{0}, 0\right)$ be Lipschitz w.r.t. $\omega$ and assume also that for every $d \in S(L)$, each $\lambda \in S^{*}(D)$ and every $\hat{\omega} \in \operatorname{supp}(\lambda)$, one has

$$
\left(H_{\hat{\omega}}\right) \quad \exists V \in \mathcal{N}(\hat{\omega}), \exists r>0 \text { s.t. } \inf _{\omega \in V} \Xi(d, u)_{\omega}=\inf _{\omega \in B(\hat{\omega}, r u)} \Xi(d, u)_{\omega}+o(1),
$$

with o(1) converging to 0 uniformly as $u \downarrow 0$. Then

$$
v_{-}^{\prime \prime}(0) \geq \inf _{d \in S(L)} \max _{\lambda \in S(D)}\left\{\mathcal{L}^{\prime \prime}\left(x_{0}, \lambda, 0\right)(d, 1)(d, 1)+\int_{\Omega} \bar{\tau}_{d}(\omega) d \lambda(\omega)\right\}
$$


where

$$
\bar{\tau}_{d}(\omega):=\mathrm{e}-\limsup _{u \downarrow 0} \Xi(d, u)_{\omega} .
$$

For $\int_{\Omega} \bar{\tau}_{d}(\omega) d \lambda(\omega)$ we adopt the same convention as in $\S 3$ : its value is the usual integral when $\bar{\tau}_{d}>-\infty$, and $+\infty$ when $\bar{\tau}_{d}$ takes the value $-\infty$. With this convention

$$
\int_{\Omega} \bar{\tau}_{d}(\omega) d \lambda(\omega)=-\sigma\left(\lambda,\left\{h \in C(\Omega) ; h+\bar{\tau}_{d} \geq 0\right\}\right),
$$

which is an u.s.c. function of $\lambda \in S(D)$.

The function $-\bar{\tau}_{d}$ may be interpreted as a lower-curvature function.

We discuss some consequences of this Proposition, postponing the proof until the end of the section.

Comparing the bounds obtained for $v_{+}^{\prime \prime}(0)$ and $v_{-}^{\prime \prime}(0)$ in Propositions 3.2 and 5.2, we see that the only difference is between the terms e-liminf $\operatorname{in}_{u 0} \Xi(d, u)$ and e-limsup $\sup _{t \downarrow 0} \Xi(d, u)$. The statement below follows.

Corollary 5.3. In addition to the assumptions of Proposition 5.2, let us suppose that $\Xi(d, u)$ epiconverges on $\operatorname{supp}(\lambda)$, for each $\lambda \in S^{*}(D)$ and $d \in S(L)$. Then there exists

$$
v^{\prime \prime}(0)=\inf _{d \in S(L)} \max _{\lambda \in S(D)}\left\{\mathcal{L}^{\prime \prime}\left(x_{0}, \lambda, 0\right)+\int_{\Omega} \mathrm{e}-\lim _{u \downarrow 0} \Xi(d, u)_{\omega} d \lambda(\omega)\right\} .
$$

It remains to find sufficient conditions to ensure the technical assumption $\left(H_{\hat{\omega}}\right)$ in Proposition 5.2. Lemma 5.5 below gives a result in this direction. We first need a technical Lemma which describes the structure of extreme points of $S(D)$.

Lemma 5.4. Suppose $(D C Q)$ and $\Lambda_{0} \neq \emptyset$. Then $S(D)$ is the closed convex hull of $S^{*}(D)$, and any $\lambda \in S^{*}(D)$ is of the form

$$
\lambda=\sum_{i=1}^{p} \lambda_{\omega_{i}} \delta_{\omega_{i}}
$$

with $p \leq n, \lambda_{\omega_{i}}<0, \delta_{\omega_{i}}$ being the Dirac mass at $\omega_{i}$ (recall that $n$ is the dimension of the space to which belongs $x$ ).

Proof. By Lemma 2.2, the set $S(D)$ is nonempty and bounded. Being closed, it is weak* compact. The Krein-Milman theorem implies that $S(D)$ is the closed convex hull of its extreme points (see e.g. [22]). Now, $S(D)$ is a face of $\Lambda_{0}$ so that the points in $S^{*}(D)$ are also extreme points of $\Lambda_{0}$, and the latter are known to be the sum of at most $n$ Dirac masses (see [20]). 
Lemma 5.5. Suppose $G\left(x_{0}, 0\right), G^{\prime}\left(x_{0}, 0\right)$ and $G^{\prime \prime}\left(x_{0}, 0\right)$ are Lipschitz w.r.t. $\omega$ and $(Q G C)$ holds. Under each of the following conditions

(i) $Z_{0}$ is a finite set,

(ii) $\Omega$ is an interval and $Z_{0}$ is the union of finitely many intervals,

property $\left(H_{\hat{\omega}}\right)$ is satisfied for all $\hat{\omega} \in \operatorname{supp}(\lambda), \forall \lambda \in S^{*}(D), \forall d \in S(L)$.

Proof. By the previous Lemma, $\lambda \in S^{*}(D)$ has a finite support included in $Z_{0}$. Then, using $d \in S(L)$, we obtain

$$
G\left(x_{0}, 0\right)_{\hat{\omega}}=G^{\prime}\left(x_{0}, 0\right)(d, 1)_{\hat{\omega}}=0, \quad \forall \hat{\omega} \in \operatorname{supp}(\lambda) .
$$

From this we deduce that for all $V \in \mathcal{N}(\hat{\omega})$ we have for all $r>0, u>0$ small enough

$$
\inf _{\omega \in V} \Xi(d, u)_{\omega} \leq \inf _{\omega \in B(\hat{\omega}, r u)} \Xi(d, u)_{\omega} \leq \Xi(d, u)_{\hat{\omega}}=0 .
$$

Let $V$ be a closed neighborhood of $\hat{\omega}$ such that $\operatorname{dist}\left(\omega, Z_{0}\right)=d(\omega, \hat{\omega})$ whenever $\omega \in V \backslash Z_{0}$ : this is possible by $(i)$ or $(i i)$. Let us consider a Lipschitz constant $L$ for

$$
g(\omega):=G^{\prime}(x, 0)(d, 1)_{\omega}
$$

and let $\omega_{u}$ minimize $\Xi(d, u)_{\omega}$ over $\omega \in V$. If $\omega_{u} \in Z_{0}$ then we get $\Xi(d, u)_{\omega_{u}} \geq 0$, which combined with (13) gives $\left(H_{\hat{\omega}}\right)$ with $o(1) \equiv 0$ and $r>0$ arbitrary. Let us then assume that $\omega_{u} \notin Z_{0}$. Then we may use $(Q G C)$ and (13) to obtain

$$
c \operatorname{dist}\left(\omega_{u}, Z_{0}\right)^{2}+u g\left(\omega_{u}\right) \leq G\left(x_{0}, 0\right)_{\omega_{u}}+u g\left(\omega_{u}\right)=\frac{u^{2}}{2} \Xi(d, u)_{\omega_{u}} \leq \frac{u^{2}}{2} \Xi(d, u)_{\hat{\omega}}=u g(\hat{\omega}) .
$$

As $g(\omega)$ is Lipschitzian, we get

$$
c d\left(\omega_{u}, \hat{\omega}\right)^{2} \leq u\left(g(\hat{\omega})-g\left(\omega_{u}\right)\right) \leq u L d\left(\omega_{u}, \hat{\omega}\right),
$$

and then $d\left(\omega_{u}, \hat{\omega}\right) \leq u L / c$. This proves that minimizing $\Xi(d, u)_{\omega}$ over $\omega \in V$ is equivalent to minimizing it over $B(\hat{\omega}, u L / c)$, proving $\left(H_{\hat{\omega}}\right)$ with $r=L / c$ and $o(1) \equiv 0$. $\square$

We now come back to the proof of Proposition 5.2, starting with the following Lemma, that does not use the specific properties of semi-infinite optimization.

Lemma 5.6. Assume (DCQ) and suppose that there exists an o( $\left.u^{2}\right)$-optimal path such that $x_{u}=x_{0}+O(u)$. Then there exists $u_{k} \downarrow 0$ and $d \in S(L)$ such that for any $\lambda \in S(D)$

$$
v_{-}^{\prime \prime}(0)=\mathcal{L}^{\prime \prime}\left(x_{0}, \lambda, 0\right)(d, 1)(d, 1)-\lim _{k \rightarrow \infty} \int_{\Omega} \Psi_{G}\left(z^{k}, d\right)_{\omega} d \lambda(\omega),
$$

where

$$
z^{k}:=\frac{x_{u_{k}}-x_{0}-u_{k} d}{u_{k}^{2} / 2}
$$


Proof. Let us take a sequence $u_{k} \downarrow 0$ such that

$$
2\left(v\left(u_{k}\right)-v(0)-u_{k} v(L)\right) / u_{k}^{2} \rightarrow v_{-}^{\prime \prime}(0) .
$$

Denoting $x^{k}=x_{u_{k}}$ and passing to a subsequence we may also assume that

$$
\left(x^{k}-x_{0}\right) / u_{k} \rightarrow d
$$

for some $d \in \mathbb{R}^{n}$ which, by [4, Proposition 3.3], satisfies $d \in S(L)$.

Since $x_{u}=x_{0}+O(u)$, a second order expansion of $G$ gives

$$
G\left(x^{k}, u_{k}\right)=G\left(x_{0}, 0\right)+u_{k} G^{\prime}\left(x_{0}, 0\right)(d, 1)+\frac{u_{k}^{2}}{2} \Psi_{G}\left(z^{k}, d\right)+o\left(u_{k}^{2}\right) .
$$

As $d \in S(L)$ and $\lambda \in S(D)$ we have

$$
\left\langle\lambda, G\left(x_{0}, 0\right)\right\rangle=\left\langle\lambda, G^{\prime}\left(x_{0}, 0\right)(d, 1)\right\rangle=0,
$$

from which we get

$$
\left\langle\lambda, G\left(x^{k}, u_{k}\right)\right\rangle=\frac{u_{k}^{2}}{2}\left\langle\lambda, \Psi_{G}\left(z^{k}, d\right)\right\rangle+o\left(u_{k}^{2}\right) .
$$

Taking into account that $\mathcal{L}\left(x_{0}, \lambda, 0\right)=v(0), \mathcal{L}_{x}^{\prime}\left(x_{0}, \lambda, 0\right)=0$, and $\mathcal{L}_{u}^{\prime}\left(x_{0}, \lambda, 0\right)=v^{\prime}(0)$, we deduce

$$
\begin{aligned}
v\left(u_{k}\right) & =f\left(x^{k}, u_{k}\right)+o\left(u_{k}^{2}\right)=\mathcal{L}\left(x^{k}, \lambda, u_{k}\right)-\left\langle\lambda, G\left(x^{k}, u_{k}\right)\right\rangle+o\left(u_{k}^{2}\right), \\
& =v(0)+u_{k} v^{\prime}(0)+\frac{u_{k}^{2}}{2}\left[\mathcal{L}^{\prime \prime}\left(x_{0}, \lambda, 0\right)(d, 1)(d, 1)-\left\langle\lambda, \Psi_{G}\left(z^{k}, d\right)\right\rangle\right]+o\left(u_{k}^{2}\right)
\end{aligned}
$$

from which the conclusion follows.

In order to get the best lower estimate, and reminding that the multiplier is nonpositive, we must minorize $\Psi_{G}\left(z^{k}, d\right)$ efficiently. Note that expanding $G\left(x^{k}, u_{k}\right)$, we get the following relation that we shall use later

$$
\Psi_{G}\left(z^{k}, d\right)+\Xi\left(d, u^{k}\right) \geq o(1)
$$

where the inequality is to be understood in $C(\Omega)$, i.e. $o(1) \rightarrow 0$ uniformly when $u \downarrow 0$.

We now proceed with the proof of Proposition 5.2.

Proof. Let $u_{k}, d, z^{k}$ be chosen as in Lemma 5.6. Consider the problem

$$
\max _{\lambda \in S(D)}\left\{\mathcal{L}^{\prime \prime}\left(x_{0}, \lambda, 0\right)(d, 1)(d, 1)+\int_{\Omega} \bar{\tau}_{d}(\omega) d \lambda(\omega)\right\} .
$$

As the cost function is affine, u.s.c. and $S(D)$ is weak* compact, the maximum is attained at an extreme point $\lambda^{*} \in S^{*}(D)$. By Lemma 5.4, $\lambda^{*}=\sum_{i=1}^{p} \lambda_{\omega_{i}} \delta_{\omega_{i}}$. 
Let us take $\hat{\omega}=w_{i}$. From (18) we have

$$
\Psi_{G}\left(z^{k}, d\right)_{\hat{\omega}}+\Xi\left(d, u_{k}\right)_{\omega} \geq \Psi_{G}\left(z^{k}, d\right)_{\hat{\omega}}-\Psi_{G}\left(z^{k}, d\right)_{\omega}+o(1),
$$

with $o(1)$ uniform w.r.t. $\omega$. Minimize the right hand side first, and then the left hand side for $\omega \in B\left(\hat{\omega}, r u_{k}\right)$, to obtain

$$
\Psi_{G}\left(z^{k}, d\right)_{\hat{\omega}}+\inf _{\omega \in B\left(\hat{\omega}, r u_{k}\right)} \Xi\left(d, u_{k}\right)_{\omega} \geq \Psi_{G}\left(z^{k}, d\right)_{\hat{\omega}}-\sup _{\omega \in B\left(\hat{\omega}, r u_{k}\right)} \Psi_{G}\left(z^{k}, d\right)_{\omega}+o(1) .
$$

Now, if we fix $r>0$, as $G^{\prime}\left(x_{0}, 0\right)$ and $G^{\prime \prime}\left(x_{0}, 0\right)$ are Lipschitzian, using the fact that $u_{k} z^{k} \rightarrow 0$, we get

$$
\Psi_{G}\left(z^{k}, d\right)_{\hat{\omega}}-\sup _{\omega \in B\left(\hat{\omega}, r u_{k}\right)} \Psi_{G}\left(z^{k}, d\right)_{\omega} \geq O\left(u_{k}\right)\left\|z^{k}\right\|+o(1)=o(1),
$$

which combined with the previous estimate gives

$$
\Psi_{G}\left(z^{k}, d\right)_{\hat{\omega}}+\inf _{\omega \in B\left(\hat{\omega}, r u_{k}\right)} \Xi\left(d, u_{k}\right)_{\omega} \geq o(1) .
$$

Invoking assumption $\left(H_{\hat{\omega}}\right)$, we may select $V \in \mathcal{N}(\hat{\omega}), r>0$ such that

$$
\liminf _{k \rightarrow+\infty} \Psi_{G}\left(z^{k}, d\right)_{\hat{\omega}}+\limsup _{u \downarrow 0} \inf _{\omega \in V} \Xi(d, u)_{\omega} \geq 0
$$

and therefore, by (10)

$$
\liminf _{k \rightarrow+\infty} \Psi_{G}\left(z^{k}, d\right)_{\hat{\omega}}+\bar{\tau}_{d}(\hat{\omega}) \geq 0 .
$$

Combining this estimate with (14), and noting that $\lambda_{\omega_{i}}<0$, we obtain (11) as required. $\square$

6. Conclusion. Let us assume that the original problem $\left(P_{0}\right)$ has a unique solution

$$
S(0)=\left\{x_{0}\right\}
$$

and that we have the uniform boundedness of solutions:

$$
\exists r>0, u_{0}>0 \text { such that for all } u \leq u_{0}, S(u) \neq \emptyset \text { and } S(u) \subset B(0, r) .
$$

Theorem 6.1. (Main Result) Let us assume $(H 1),(H 2),(D C Q),(Q G C),(S O C)$ and $S(L) \neq \emptyset$. Suppose also that $G\left(x_{0}, 0\right), G^{\prime}\left(x_{0}, 0\right)$ and $G^{\prime \prime}\left(x_{0}, 0\right)$ are Lipschitz w.r.t. $\omega$.

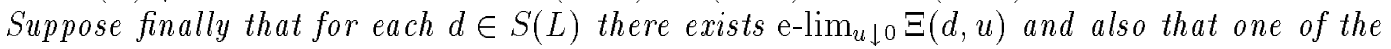
following conditions hold

(i) $Z_{0}$ is finite,

(ii) $\Omega$ is an interval and $Z_{0}$ is the union of finitely many intervals.

Then we have 
(a) The value function has first and second order (right) derivatives given by $v^{\prime}(0)=$ $v(L), v^{\prime \prime}(0)=v(Q)$. Moreover, if $v(Q)>-\infty$ we have the expansion

$$
v(u)=v(0)+u v(L)+\frac{u^{2}}{2} v(Q)+o\left(u^{2}\right) .
$$

(b) The set of all limit points of $\left(x_{u}-x_{0}\right) / u$, where $x_{u}$ ranges over all paths of o( $\left.u^{2}\right)$ optimal solutions, is included in $S(Q)$. In particular, if $S(Q)$ is a singleton, i.e. $S(Q)=\{d\}$, and $x_{u}$ is as above, then $x_{u}=x_{0}+u d+o(u)$.

(c) Let $d \in S(Q)$. If there exists $z \in S\left(L_{d}\right)$ (this is always the case when (i) holds) then there exists an $o\left(u^{2}\right)$-optimal path $x_{u}=x_{0}+u d+o(u)$.

(d) Let $\lambda_{u}$ be a multiplier associated with a solution $x_{u}$ of $\left(P_{u}\right)$. Then all weak limit points of $\lambda_{u}$ belong to $S(D)$.

Proof. From $(H 1),(H 2)$ there exists $x_{u} \in S(u)$ which satisfies $x_{u} \rightarrow x_{0}$ when $u \downarrow 0$. Then, from Proposition 4.1 we get $x_{u}=x_{0}+O(u)$, and part (a) follows by combining Lemmas 5.1 and 5.5 with Corollary 5.3.

If $x_{u}$ is a path of $o\left(u^{2}\right)$-optimal solutions, expanding $f\left(x_{u}, u\right)$ as in the proof of Lemma 5.6 , we obtain the first statement in $(b)$. The second statement is an immediate consequence of the first.

We now prove (c). Let $d \in S(Q)$ and $z \in S\left(L_{d}\right)$. Then there exists a feasible path $x_{u}=x_{0}+u d+\frac{u^{2}}{2} z+o\left(u^{2}\right)$. Expanding $f\left(x_{u}, u\right)$ and $G\left(x_{u}, u\right)$, we obtain $v(Q)=\Psi_{f}(z, d)$ as well as (2). The conclusion follows.

Assertion (d) is a consequence of [4, Proposition 3.3].

Concluding remarks. Our final result is an extension to semi-infinite optimization of the results of the sequence of papers [8], [19], [2] and [6], in the following sense: if $\Omega$ is a finite set, then we recover exactly the above mentioned results, up to the presence of equality constraints. However, there is no difficulty in adding a finite number of equality constraints to our formulation. We avoided it for the sake of clarity of exposition, and in order to concentrate on the real difficulty, which is to handle an infinite number of constraints.

Some of our hypotheses, however, may seem unduly strong. First of all, we assume $S(L)$ to be non empty. While this hypothesis is automatically satisfied when the contact set is finite (due to the standard theory of linear programming) we are not aware of general criteria allowing to check nonemptyness of $S(L)$ for semi-infinite programming. Performing an analysis of the variation of the solutions when $S(L)$ is empty is an open problem. Some of the results of part II might be useful for dealing with this case.

The other hypothesis that seems excessively strong is the alternative ( $i)$ or ( $i i)$. We need it in order to satisfy the geometrical hypothesis $\left(H_{\hat{\omega}}\right)$. Still, the most important contribution of this paper is to present a new way of obtaining sharp under-estimates of the cost, and we hope that the technique presented here can be improved in order to deal with more general contact sets. 
[1] H. Attouch, Variational convergences for functions and operators, Pitman Advanced Publishing Program (1984).

[2] A. Auslender and R. Cominetti, First and second order sensitivity analysis of nonlinear programs under directional constraint qualification conditions, Optimization 21(1990), pp. 351-363.

[3] A. Ben-Tal, M. Teboulle and J. Zowe, Second order necessary optimality conditions for semi-infinite programming problems, Lecture notes in control and information sciences 15, R. Hettich ed., Springer Verlag, Berlin, 1979, pp. 17-30

[4] J.F. Bonnans and R. Cominetti, Perturbed optimization in Banach spaces $I$ : a general theory based on a weak directional constraint qualification, Rapport de Recherche INRIA n 2024 (1993); SIAM J. Control Optimization, to appear.

[5] J.F. Bonnans and R. Cominetti, Perturbed optimization in Banach spaces II : a theory based on a strong directional qualification, Rapport de Recherche INRIA n ${ }^{\circ} 2161$ (1994); SIAM J. Control Optimization, to appear.

[6] J.F. Bonnans, A.D. Ioffe and A. Shapiro, Expansion of exact and approximate solutions in nonlinear programming, in Proc. French-German Conference in Optimization, D. Pallaschke ed., Lecture Notes in Economics and Math. Systems, Springer-Verlag (1992).

[7] R. Cominettiand J.P. Penot, Tangent sets of order one and two to the positive cones of some functional spaces, Preprint, 1993.

[8] J. Gauvin and R. Janin, Directional behavior of optimal solutions in nonlinear mathematical programming, Math. Oper. Res. 13(1988), pp. 629-649.

[9] R. Hettich and K.O. Kortanek, Semi-infinite programming: theory, methods, and applications, SIAM Review 35(1994), pp. 380-429

[10] A.D. Ioffe, On sensitivity analysis of nonlinear programs in Banach spaces: the approach via composite unconstrained optimization, SIAM J. Optimization 4(1994), pp. 1-43.

[11] A.D. Ioffe, Second order conditions in nonlinear nonsmooth problems of semi-infinite programming, in Lecture notes in control and information sciences 215, A.V. Fiacco and K.O. Kortanek eds., Springer Verlag, Berlin, 1983, pp. 262-280

[12] H.T. Jongen, F. Twilt, and G.W. Weber, Semi-infinite optimization : structure and stability of the feasible set, J. Optim. Theory Appl. 72(1992), pp. 529-552

[13] D. Klatte,, Stability of stationary solutions in semi-infinite optimization via the reduction approach, in Lecture notes in economics and mathematical systems 382, W. Oettli and D. Pallaschke eds., Springer Verlag, Berlin, 1992, pp. 155-170

[14] H. Maurer and J. Zowe, First and second order necessary and sufficient optimality conditions for infinite dimensional programming problems, Math. Prog., 16(1979), pp. 98-110.

[15] J.P. Penot, Optimality conditions for minimax problems, semi-infinite programming problems and their relatives. Report 92/16, UPRA, Laboratoire de Math. Appl., Av. de l'Université, 64000 Pau, France.

[16] E. Polak, On the mathematical foundations of nondifferentiable optimization in engineering design, SIAM Review 29(1987), pp. 21-89

[17] D. Torralba, An epigraphical calculus for quasi-monotone sequences of functions, Dept of Mathematics, University of Montpellier II, Place Eugène Bataillon, France. Submitted paper (1993).

[18] A. Shapiro, Second-order derivatives of extremal-value functions and optimality conditions for semiinfinite programming, Math. Oper. Res. 10(1985), pp. 207-219

[19] A. Shapiro, Sensitivity analysis of nonlinear programs and differentiability properties of metric projections, SIAM J. Control \& Opt. 26(1988), pp. 628-645.

[20] A. Shapiro, On Lipschitzian stability of optimal solutions of parametrized semi-infinite programs. Math. Oper. Research, to appear.

[21] A. Shapiro, Directional differentiability of the optimal value function in convex semi-infinite programming, to appear.

[22] K. Yosida, Functional analysis, Springer-Verlag, Berlin, 1980.

[23] J. Zowe and Kurczyusz, Regularity and stability for the mathematical programming problem in Banach spaces, Applied Math. and Optimization 5(79), pp. 49-62. 
Unité de recherche INRIA Lorraine, Technopôle de Nancy-Brabois, Campus scientifique, 615 rue du Jardin Botanique, BP 101, 54600 VILLERS LES NANCY

Unité de recherche INRIA Rennes, Irisa, Campus universitaire de Beaulieu, 35042 RENNES Cedex Unité de recherche INRIA Rhône-Alpes, 46 avenue Félix Viallet, 38031 GRENOBLE Cedex 1

Unité de recherche INRIA Rocquencourt, Domaine de Voluceau, Rocquencourt, BP 105, 78153 LE CHESNAY Cedex

Unité de recherche INRIA Sophia-Antipolis, 2004 route des Lucioles, BP 93, 06902 SOPHIA-ANTIPOLIS Cedex

\section{Éditeur}

INRIA, Domaine de Voluceau, Rocquencourt, BP 105, 78153 LE CHESNAY Cedex (France)

ISSN 0249-6399 